Journal of Physics and Its Applications

Journal homepage : https://ejournal2.undip.ac.id/index.php/jpa/index

\title{
The comparison of size-specific dose estimate in CT examination based on head and body PMMA phantom
}

\author{
Mohd Hanafi Ali ${ }^{1}$, Choirul Anam ${ }^{2}$, Freddy Haryanto ${ }^{3}$, and Geoff Dougherty 4 \\ ${ }_{1}$ Department of Medical Radiation Sciences, The University of Sydney, Sydney, Australia. Email: hanafi.ali@sydney.edu.au \\ ${ }_{2}$ Department of Physics, Faculty of Mathematics and Natural Sciences, Diponegoro University, Tembalang, Semarang, Central Java, Indonesia \\ ${ }_{3}$ Department of Physics, Faculty of Mathematics and Natural Sciences, Bandung Institute of Technology, Bandung, West Java, Indonesia \\ ${ }_{4}$ Applied Physics and Medical Imaging, California State University Channel Islands, Camarillo, CA, USA
}

\section{A R T I C LE I N F O}

Article history:

Received : September 2018

Accepted : November 2018

Keywords:

$\mathrm{CTDI}_{\mathrm{vol}}$

SSDE

Water-equivalent diameter

Patient dose

\begin{abstract}
A B S T R A C T
Nowadays, a dose estimate for individual patients undergoing CT examination is carried out using the metric of size-specific dose estimate (SSDE), which is calculated by multiplying a volume CT dose index ( $\mathrm{CTDI}_{\mathrm{vol}}$ ) and a correction factor that is a function of patient size. Two $\mathrm{CTDI}_{\mathrm{vol}}$ values are based on head and body PMMA phantoms. There are also two values of correction factors $(k)$, both for head and body PMMA phantoms. The purpose of this study was to compare the SSDE values calculated using head and body PMMA phantoms

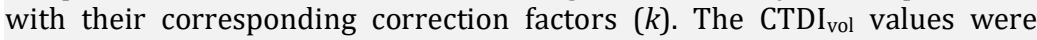
derived from the ImPACT 1.04 software for 12 CT scanners: Sensation 4, Sensation 16, Sensation 64, Light Speed, Light Speed 16, Light Speed VCT, Secura, Brilliance 16, Brilliance 64, Asteion Dual, Aquilion 4, and Aquilion 16. The size of the patients who underwent CT examination was characterized by a water-equivalent diameter $\left(\mathrm{D}_{\mathrm{w}}\right)$ from $10 \mathrm{~cm}$ to $45 \mathrm{~cm}$. The results indicated that the differences in SSDE values based on head and body CTDI $_{\text {vol }}$ were within $20 \%$. Thus, the SSDE value can be calculated using the head or body $\mathrm{CTDI}_{\text {vol }}$ bases with corresponding $k$ value.
\end{abstract}

\section{Introduction}

For about three decades, the dose from CT scanners was quantified using the CT dose index (CTDI), which is measured using a standard PMMA phantom with a diameter of either $16 \mathrm{~cm}$ or $32 \mathrm{~cm}[1,2]$. There are several derivatives of CTDI, namely, CTDIF $_{D A}$, CTDI $_{100}$, $\mathrm{CTDI}_{p}, \mathrm{CTDI}_{c}, \mathrm{CTDI}_{\mathrm{w}}$, and $\mathrm{CTDI}_{\mathrm{vol}}[3,4]$. As a dose index, CTDI is very useful for quality control [5], accreditation [6], and comparing the dose level of different CT scanners [7]. However, CTDI is a dose index and is not meant to estimate the dose received by a patient $[8,9]$. The discrepancy between CTDI and the dose received by patients can vary by more than $100 \%$, depending on the specific characteristics of the patient $[10,11]$. Studies have shown that for fixed setting parameters $(\mathrm{kVp}, \mathrm{mAs}$, pitch, etc.), the patient dose is highly associated with patient size $[12,13]$, weight $[14,15]$, or body mass index $[16,17]$. In 2011, the AAPM issued report No. 204 on pediatric dose estimation in CT, taking into account the patient's size, and introduced the size-specific dose estimate (SSDE) as a new descriptor [18]. The SSDE relies on a volume CTDI (CTDI $\left.{ }_{\mathrm{vol}}\right)$ and is corrected by a correction factor based on patient size [19-21].

The SSDE value depends on a number of factors. It is calculated as $\mathrm{CTDI}_{\mathrm{vol}} \times k(\mathrm{D})$. Thus, the accuracy of SSDE is strongly influenced by three factors, namely, size of the patient (D), correction factor $(k)$, and $\mathrm{CTDI}_{\mathrm{vol}}$ value. In AAPM reportNo. 204, the size of the patient was characterized by the effectivediameter ( $D_{\text {eff }}$ ) [18], which could be calculated fromthe cross-section of patients. However, for practical considerations, the $D_{\text {eff }}$ is estimated using only a lateral diameter, anteriorposterior diameter, or a combination of both $[19,22]$. The $D_{\text {eff }}$ can also be estimated from the patient's age, but its accuracy will be rather low [23]. However, the $D_{\text {eff }}$ only takes into account the size and neglects the composition or attenuation of the patient. In reality, a significant amount of real dose is determined by the composition of the body part being scanned [24, 25]. In order to obtain a more accurate dose estimate, a water-equivalent diameter $\left(D_{w}\right)$ was introduced in lieu of the $D_{\text {eff. }}$ The $D_{w}$ was previously introduced by Wang et al [26] and adopted by AAPM in report No. 220 [27]. The accuracy of SSDE also depends on how the $D_{\text {eff }}$ and $D_{w}$ are calculated [28-30].

The correction factor $(k)$ in the AAPM report No. 204 was obtained by combining data from four different research groups that used different methods and different types of scanners [18]. All data were then combined to determine the values of the correction factor for various values of patient diameters. The data from the four different studies were highly correlated with a correlation coefficient $\left(\mathrm{R}^{2}\right)$ of 0.942 . The mean deviation between these four groups was $3.3 \%$, and the maximum difference was $+16 \%$ for the smallest patient $(12.2 \mathrm{~cm})$ [18]. These results allowed the creation of a single graph 
of $k$ based on the head PMMA phantom and a singlegraph of $\mathrm{k}$ based on the body PMMA phantom. The $k$ value may be applied with acceptable accuracy to all types of scanners and all CT centers $[18,31]$.

The $\mathrm{CTDI}_{\mathrm{vo}} \mathrm{l}$ value determines the SSDE. Two values of $\mathrm{CTDI}_{\mathrm{vol}}$ are measured on the $16 \mathrm{~cm}$ (head) and $32 \mathrm{~cm}$ (body) PMMA phantoms. Currently, there is no standard for the phantom diameter that should be used for pediatric protocols or when the small or medium acquisition field of view (FOV) is selected [32]. For that purpose, Siemens and Philips use the body PMMA phantom, but GE and Toshiba use the head PMMA phantom [32]. The use of either was expected to produce the comparable SSDE value. Up to now, the comparison of SSDE based on the head and body PMMA phantom has not been accomplished. Therefore, this study sought to evaluate the comparison of SSDE values based on the head and body PMMA phantom for 12 different scanners.

\section{Methods}

\subsection{Water-equivalent diameter}

The $\mathrm{D}_{\mathrm{w}}$ was originally introduced by Huda et al [15]. However, they did not actually compare the dose of the scanned object and $D_{w}$. In 2011, Wang et al provided a mathematical description for the $D_{w}$ and investigated its relationship with individual patient doses [26]. The current study did not intend to evaluate the feasibility of $D_{w}$ or the method for calculating it, because this metric has been evaluated and the method for calculating it has already been established $[24,26]$. Previous studies have shown that the use of $D_{w}$ was more appropriate to describe the size of patients, especially for the thoracic region of the body, because the $\mathrm{D}_{\mathrm{w}}$ considers not only the size but also the attenuation of the body part. In the current study, we used a $D_{w}$ range from $10 \mathrm{~cm}$ to 45 $\mathrm{cm}$, which covered patient sizes from newborns to very obese adult patients.

\subsection{CTDI $_{\text {vol }}$}

The Imaging Performance Assessment of CT scanners (ImPACT) group developed an Excel (Microsoft) spreadsheet to provide a user friendly interface for determining output CT dose and organ doses by using the National Radiological Protection Board (NRPB) Monte Carlo dose datasets [33]. The software was validated by many investigators of CT dosimetry [34-35]. In this current study, the CTDIvol values were derived from the ImPACT CT patient dosimetry version 1.04 software (released in May 2011) [33] for 12 types of scanners as listed in Table 1 . The CTDIvol values were based on two PMMA phantoms, namely the head (16 cm in diameter) and the body $(32 \mathrm{~cm}$ in

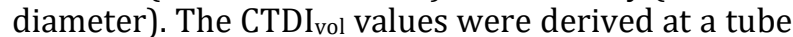
voltage of $120 \mathrm{kVp}$, tube current of $100 \mathrm{~mA}$, rotation time of $0.6 \mathrm{~s}$, and a spiral pitch of 1 . Beam collimation was set to $10 \mathrm{~mm}$ or to the value closest to $10 \mathrm{~mm}$ if it was not available (see Table 1).

\subsection{SSDE calculation}

SSDE was calculated as the product of CTDI $_{\text {vol }}$ and the correction factor as a function of patient size, $k\left(D_{\mathrm{w}}\right)$. When using the head-based CTDI $_{\text {vol, a }} k^{h}$ correction factor was used, and when using the body -based $\mathrm{CTDI}_{\mathrm{vol}}$, a $k^{b}$ correction factor was used. Therefore, the equations for calculating $\operatorname{SSDE}^{\mathrm{h}}$ and $\mathrm{SSDE}^{\mathrm{k}}$ can be written as follows:

$$
\begin{aligned}
& S S D E^{h}=C T D I_{v o l}^{h} \times k^{h} \\
& S S D E^{b}=C T D I_{v o l}^{b} \times k^{b}
\end{aligned}
$$

$k^{b}$ and $k^{h}$ values were taken from AAPM report No. 204. The graphs of $k^{h}$ and $k^{b}$ for various diameters are shown in Fig. 1.

Table 1:Type of scanners and the collimation values used in this study

\begin{tabular}{lc}
\hline \multicolumn{1}{c}{ Scanner } & Beam collimation (mm) \\
\hline Siemens & 10 \\
Sensation 4 & 10 \\
Sensation 16 & 10 \\
Sensation 64 & \\
GE & 10 \\
Light Speed & 10 \\
Light Speed 16 & 10 \\
Light Speed VCT & \\
Philips & 12 \\
Secura & 10 \\
$\quad$ Brilliance 16 & 10 \\
Brilliance 64 & \\
Toshiba & 10 \\
Asteion Dual & 12 \\
Aquilion 4 & 12 \\
Aquilion 16 &
\end{tabular}

\section{Results}

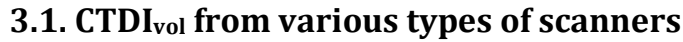

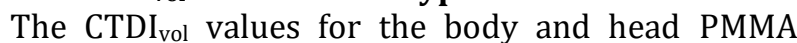
phantoms from the 12 scanners listed in the ImPACT software are shown in Fig. 2. The body CTDI $_{\text {vol values }}$ were approximately half the head $\mathrm{CTDI}_{\text {vol values. The }}$ average body $\mathrm{CTDI}_{\text {vol value was }} 5.9 \pm 1.7 \mathrm{mGy}$ and the average head $\mathrm{CTDI}_{\text {vol }}$ value was $11.9 \pm 3.2 \mathrm{mGy}$. The

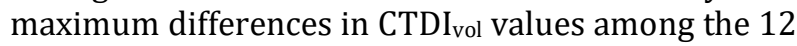
scanners were $157 \%$ for the body and $143 \%$ for the head.

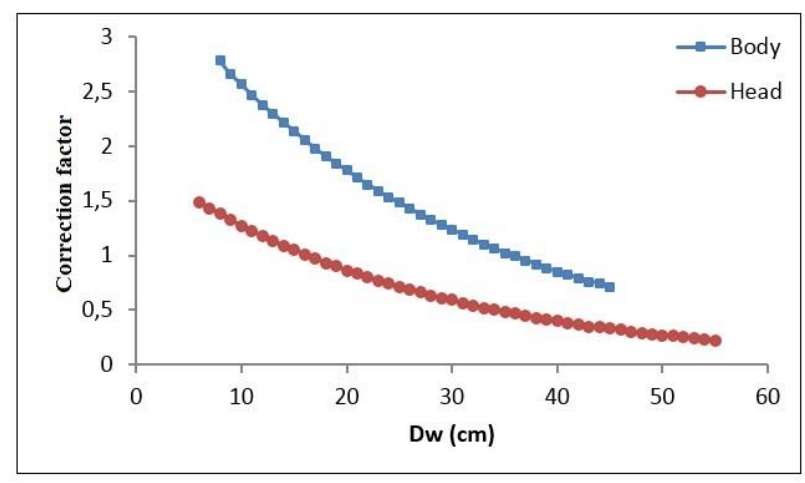

Fig. 1: The graphs plotting the correction factors $k^{b}$ and $k^{h}$ against the diameters of the patient [18] 


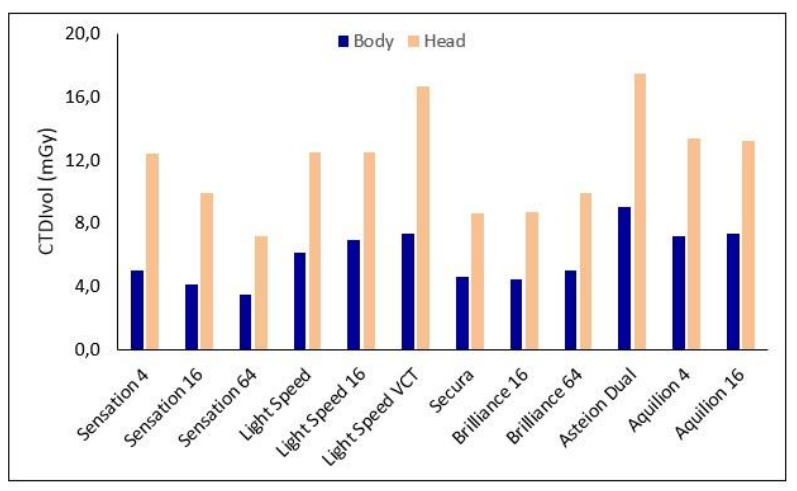

Fig. 2:The $\mathrm{CTDI}_{\mathrm{vol}}$ values for the body and head PMMA phantoms from the 12 scanners listed in the ImPACT software

\subsection{SSDE from various types of scanners}

The SSDE values for the 12 scanners are shown in Fig. 3 . The SSDE values decreased exponentially with increasing $\mathrm{D}_{\mathrm{w}}$ because the values of $k$ used to calculate the SSDE also decreased exponentially with increasing $\mathrm{D}_{\mathrm{w}}$. The SSDE values among the 12 scanners used appeared to have a maximum difference of up to $157 \%$ when calculated using the body CTDI $_{\text {vol }}$ and $143 \%$ when calculated using the head $\mathrm{CTDI}_{\mathrm{vol}}$. These differences reflect the differences in $\mathrm{CTDI}_{\mathrm{vol}}$ values as shown in Fig. 2.

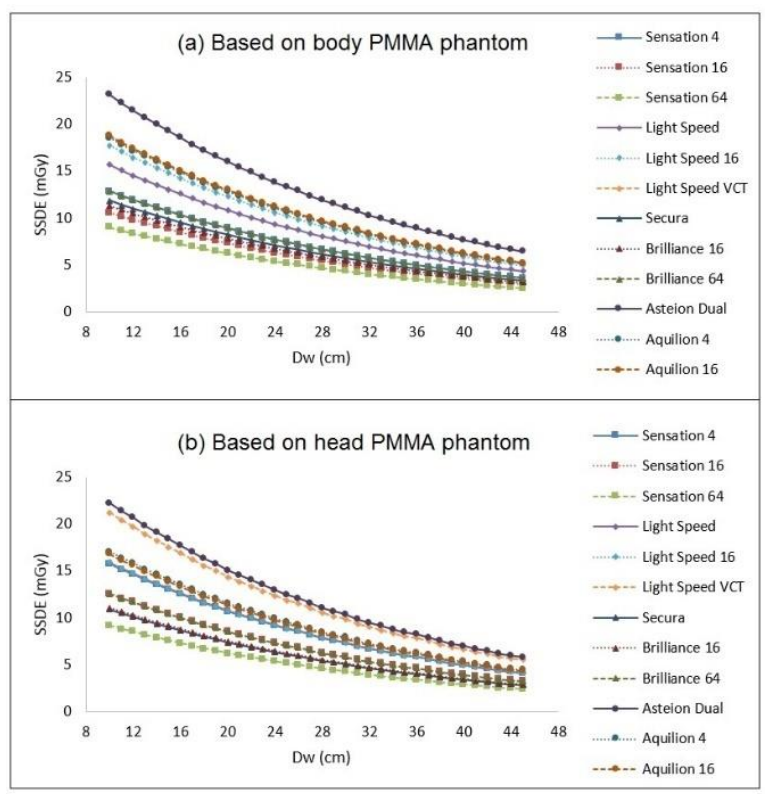

Fig. 3:SSDE versus $D_{w}$ for 12 scanners, (a) based on the body $\mathrm{CTDI}_{\mathrm{vol}}$, and (b) based on the head $\mathrm{CTDI}_{\mathrm{vol}}$

\subsection{SSDE comparison based on the body and head CTDI ${ }_{\text {vol }}$}

The SSDE values based on either the body or head $\mathrm{CTDI}_{\text {vol }}$ should be identical. However, the two may differ significantly. The comparisons of SSDE values based on the body and head CTDI $_{\text {vol }}$ for the 12 scanners are shown in Fig. 4 and Table 2. The differences between them were within 20\%. The highest absolute difference was for the Sensation 4 scanner (18.8 $\pm 2.6 \%)$, and the lowest absolute
Table 2:The difference in SSDE values based on the body and head CTDI ${ }_{\text {vol }}$ for 12 scanners

\begin{tabular}{ll}
\hline Scanner & $\begin{array}{l}\text { The difference of SSDE } \\
\text { based on body and head } \\
\text { CTDIvol (\%) }\end{array}$ \\
\hline
\end{tabular}

$\begin{array}{ll}\text { Siemens } & \\ \quad \text { Sensation } 4 & -18.8 \pm 2.6 \\ \quad \text { Sensation } 16 & -15.6 \pm 2.5 \\ \quad \text { Sensation } 64 & 1.5 \pm 2.1 \\ \text { GE } & \\ \quad \text { Light Speed } & 1.9 \pm 2.1 \\ \quad \text { Light Speed 16 } & 13.2 \pm 1.9 \\ \quad \text { Light Speed VCT } & -9.6 \pm 2.4 \\ \text { Philips } & \\ \text { Secura } & 10.5 \pm 1.9 \\ \quad \text { Brilliance 16 } & 5.3 \pm 2.0 \\ \quad \text { Brilliance 64 } & 5.2 \pm 2.0 \\ \text { Toshiba } & \\ \text { Asteion Dual } & 6.9 \pm 2.0 \\ \text { Aquilion } 4 & 10.9 \pm 1.9 \\ \text { Aquilion } 16 & 13.4 \pm 1.9\end{array}$

difference was for the Sensation 64 scanner $(1.5 \pm$ $2.1 \%)$.

\section{Discussion}

SSDE is a descriptor used to estimate the dose for an individual patient undergoing a CT examination. SSDE is designed to be as simple as possible; therefore, it can be calculated easily in the clinical setting. However, three main reasons cause the SSDE estimate to differ from the real dose, i.e., inaccuracies in the value of the correction factor $(k)$, in characterizing the individual patient (depending on the metrics and techniques used), and in the CTDI $I_{\text {vol }}$ value. SSDE is calculated on the basis of a single

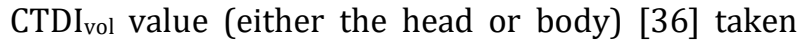
from the screen console, the DICOM header, or the dose report. In addition, $\mathrm{CTDI}_{\mathrm{vol}}$ may be measured using the pencil ionization chamber or CT dose profiler [2]. At every imaging center, the $\mathrm{CTDI}_{\mathrm{vol}}$ should be measured periodically as part of a quality control program [5]. The measurement is usually carried out using two types of PMMA phantoms, namely, the head and body phantoms.

SSDE for head examinations would be using the head CTDIvolvalue, and for thoracic, abdominal, and pelvic examinations, it would be using the body

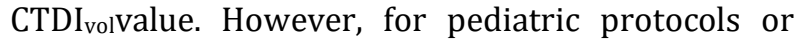
when the small FOV is selected, there is no standard for the phantom diameter that should be used. For example, The body CTDI $_{\text {vol }}$ value was used by Siemens and Philips, and on other hand GE and Toshiba use the head CTDI $I_{\text {vol }}$ value [32]. In this current study, the SSDE calculation based on both two CTDI ${ }_{\text {vol values }}$ was compared. 


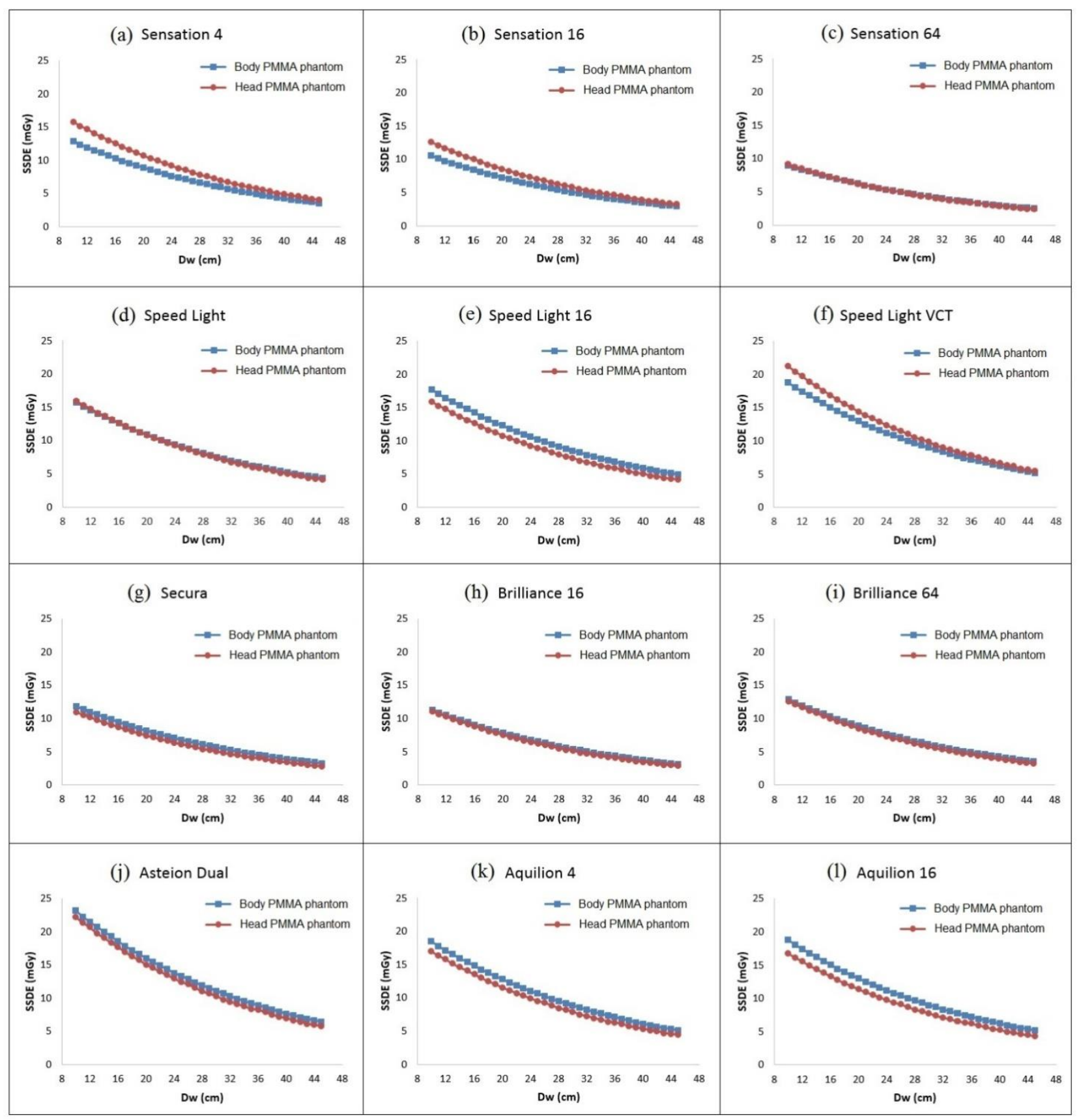

Fig. 4: The graph of SSDE based on the head and body $\mathrm{CTDI}_{\mathrm{vol}}$ versus $\mathrm{D}_{\mathrm{w}}$ for individual scanner

The finding of the current study was that the differences in SSDE values based on the head and body $\mathrm{CTDI}_{\text {vol }}$ were within $20 \%$. From the 12 scanners that were examined, six $(\sim 50 \%)$ produced a difference of more than $10 \%$. Hence, the current study showed the SSDE calculation could use eitherbody or head $\mathrm{CTDI}_{\mathrm{vol}}$ value with the corresponding $k$ value.

We found that the SSDE value for one type of scanner could not be estimated from another type of scanner, even when the scanners are from the same manufacturer or have the same number of detector arrays. The difference in SSDE values among scanners can vary more than $100 \%$ because of their different specifications. Consequently, the estimates of organ dose and effective dose must also bedetermined using the same type of scanner. The conversion factor to estimate organ doses is more flexible if it is normalized by $\mathrm{CTDI}_{\mathrm{vol}}$, as reported by Turner et al [37]. The current study was only carried out on 12 different scanners. A more extensive study should be conducted to include modern MDCT scanners with detector arrays above 64 .

\section{Conclusions}

The differences in SSDE values based on the head and body $\mathrm{CTDI}_{\mathrm{vol}}$ derived from the ImPACT software for the 12 scanners were within 20\%. Hence, the SSDE value could be calculated using the head or body $\mathrm{CTDI}_{\mathrm{vol}}$ bases with the corresponding $k$ value. 


\section{Acknowledgment}

This work was funded by the Penelitian Dasar Unggulan Perguruan Tinggi (PDUPT), Kementerian Riset, Teknologi, dan Pendidikan Tinggi, Republik Indonesia (532z/I1.C01/PL/2018). The authors would like to thank Dr. Sue Edyvean from ImPACT.

\section{References}

1. T. B.Shope, R. M. Gagne, and G. C. Johnson,"A method for describing the doses delivered by transmission x-ray computed tomography," Med. Phys. 8, 488-495 (1981).

2. C. Anam, F. Haryanto, R. Widita, I. Arif, T. Fujibuchi, T. Toyoda, and G. Dougherty,"Scatter index measurement using a CT dose profiler,"J. Med. Phys. Biop.4, 95-102 (2017).

3. W. A. Kalender,"Dose in x-ray computed tomography," Phys. Med. Biol. 59, R129-R150 (2014).

4. M. F. McNitt-Gray,"AAPM/RSNA physics tutorial for residents: topics in CT-radiation dose in CT,"RadioGraphics22, 1541-1553 (2002).

5. International Atomic Energy Agency,"Quality assurance programme for computed tomography: diagnostic and therapy applications," Human Health Series No. 19. Vienna, IAEA(2012).

6. American Association of Physicists in Medicine,"The measurement, reporting, and management of radiation dose in CT," Report No. 96. Report of the Task Group 23 of the Diagnostic Imaging Council CT Committee. College Park, MD: AAPM(2008).

7. J. Vassileva, M. M. Rehani, K. Applegate, N. A. Ahmed, H. Al-Dhuhli, and H. M. Al-Naemi,"IAEA survey of paediatric computed tomography practice in 40 countries in Asia, Europe, Latin America and Africa: procedures and protocols," Eur.Radiol. 23, 623-631 (2013).

8. C. Anam, F. Haryanto, R. Widita, and I. Arif, "Automated estimation of patient's size from 3D image ofpatient for sizespecificdoseestimates (SSDE)," Adv. Sci. Eng. Med. 7, 892-896 (2015).

9. C. H. McCollough, S. Leng, Y. Lifeng, D. D. Cody, J. M. Boone, and M. F. McNitt-Gray,"CT dose index and patient dose: they are not the same thing," Radiology 259, 311-316 (2011).

10. M. Nasir, D. Pratama, C. Anam, and F. Haryanto,"Calculation of size specific dose estimates (SSDE) value at cylindrical phantom from CBCT Varian OBI v1.4 X-ray tube EGSnrc Monte Carlo simulation based," J.Phy.Conf. Ser. 694, 012040 (2016).

11. X. Li, D. Zhang, and B. Liu,"Monte Carlo assessment of CT dose equilibration in PMMA and water cylinders with diameters from 6 to 55 cm," Med. Phys. 40, 031903 (2013).

12. E. L. Nickoloff, A. K. Dutta, and Z. F. Lu,"Influence of phantom diameter, $\mathrm{kVp}$ and scan mode upon computed tomography dose Index," Med. Phys. 30, 395-402 (2003).

13. C. Anam, F. Haryanto, R. Widita, I. Arif, G. Dougherty, andD. McLean, "Volume computed- tomography dose index (CTDI $\left.{ }_{\text {vol }}\right)$ and size-specific dose estimate (SSDE) for tubecurrent modulation (TCM) in CT scanning,"Int. J.Radiat. Res.16, 289297 (2018).

14. T. Fearon, H. Xie, J. Y. Cheng, H. Ning, Y. Zhuge, and R. W. Miller,"Patient-specific CT dosimetry calculation: a feasibility study," J.Appl.Clin. Med. Phys. 12, 196-209 (2011).

15. W. Huda, E. M. Scalzetti, andM. Roskopf,"Effective doses to patients undergoing thoracic computed tomography examinations," Med. Phys. 27, 838844 (2000).

16. T. Fujibuchi, N. Funabashi, M. Hashimoto, H. Kato, M. Kurokawa, H. M. Deloar, E. Kunieda, I. Komuro, and T. Sakae,"Estimate of organ radiation absorbed dose in clinical CT using the radiation treatment planning system,"Radiat.Prot.Dosim. 142, 174-183 (2010).

17. K. Perisinakis, A. Tzedakis, and J. Damilakis,“On the use of Monte Carlo-derived dosimetric data in the estimation of patient dose from CT examinations," Med. Phys. 35, 2018-2028 (2008).

18. American Association of Physicists in Medicine,"Size-specific dose estimates (SSDE) in pediatric and adult body CT examinations," Report No. 204. Report of the Task Group 204. College Park, MD: AAPM (2011).

19. C. Anam, F. Haryanto, R. Widita, I. Arif, and G. Dougherty, "A fully automated calculation of sizespecific dose estimates (SSDE) in thoracic and head CT examinations," J. Phy. Conf. Ser. 694, 012030 (2016).

20. C. Anam, F. Haryanto, R. Widita, I. Arif, andG. Dougherty,"The evaluation of the effective diameter $\left(D_{\text {eff }}\right)$ calculation and its impact on the size-specificdose estimate (SSDE)," Atom Indonesia,43, 55-60 (2017).

21. C. Anam, F. Haryanto, R. Widita, I. Arif, and G. Dougherty, "Dose-volume product (DVP) as descriptor for estimating total energy imparted to patient undergoing CT examination," J. Med. Phys. Biop. 3, 45-55 (2016).

22. K. J. Strauss andM. J. Goske, "Estimated pediatric radiation dose during CT,"Pediatr.Radiol. 41, S472-S482 (2011).

23. J. A. Christner, N. N. Braun, M. C. Jacobsen, R. E. Carter, J. M. Kofler, andC. H. McCollough,"Sizespecific dose estimates for adult patients at CT of the torso, Radiology 265, 841-847 (2012).

24. C. Anam, F. Haryanto, R. Widita, I. Arif, G. Dougherty, and D. McLean,"Estimation of Eye Radiation Dose during Nasopharyngeal CT Examination for an Individual Patient," Information (Japan) 19, 3951-3962 (2016).

25. C. Anam, T. Fujibuchi, T. Toyoda, N. Sato, F. Haryanto, R. Widita, I. Arif, andG. Dougherty,"A simple method for calibrating pixel valuesof the ct localizer radiograph for calculatingwaterequivalent diameter and size-specific doseestimate,"Radiat.Prot.Dosim. 179, 158-168 (2018). 
26. J. Wang, J. A. Christner, X. Duan, S. Leng, L. Yu, and C. H. McCollough,"Attenuation-based estimation of patient size for the purpose of size specific dose estimation in CT. Part II. Implementation on abdomen and thorax phantoms using cross sectional CT images and scanned projection radiograph images," Med. Phys. 39, 6772-6778 (2012).

27. American Association of Physicists in Medicine,"Use of water equivalent diameter for calculating patient size and size-specific dose estimates (SSDE) in CT," Report No. 220. Report of the AAPM Task Group 220. College Park, MD: AAPM (2014).

28. C. Anam, F. Haryanto, R. Widita, I. Arif, G. Dougherty, and D. McLean,"The impact of patient table on size-specific dose estimate (SSDE),"Australas. Phys.Eng.Sci. Med. 40, 153 158 (2017).

29. S. Leng, M. Shiung, X. Duan, L. Yu, Y. Zhang, andC. H. McCollough, "Size-specific dose estimates in chest, abdomen, and pelvis CT: Impact of intrapatient variability in water equivalent diameter," Radiology276,184-190 (2015).

C. Anam, F. Haryanto, R. Widita, I. Arif, andG. Dougherty, "Automated calculation of waterequivalent diameter $\left(\mathrm{D}_{\mathrm{w}}\right)$ based on AAPM task group 220," J.Appl.Clin. Med. Phys. 17, 320-333 (2016).

30. T. Haba,S. Koyama, Y. Kinomura, Y. Ida, and M. Kobayashi,"Influence of 320-detector-row- volume scanning and AAPM report111 CT dosimetry metrics on size-specific dose estimate: a MonteCarlo study,"Australas. Phys.Eng.Sci. Med.39, 697-703 (2016).

31. J. A. Seibert, J. M. Boone, S. L. Wootton-Gorges, andR. Lamba,"Dose is not always what it seems: Where very misleadingvalue scan resultfrom volume CT doseindex and doselengthproduct,"J. Am. Coll. Radiol. 11, 233-237 (2014).

32. http://www.impactscan.org. Accessed October $29,2016$.

33. W. Huda, A. Sterzik, S. Tipnis, andU. J. Schoepf,"Organ doses to adult patients for chest CT," Med.Phys. 37, 842-847 (2010).

34. Y. Zhang, X. Li, W. P. Segars, and E.Samei,“Organ doses, effective doses, and risk indices in adult CT: Comparison off our types of reference phantoms across different examination protocols," Med. Phys. 39, 3404-3423 (2012).

35. C. Anam, F. Haryanto, R. Widita, I. Arif, and G. Dougherty, "The size-specific dose estimate (SSDE) for truncated computed tomography images,"Radiat.Prot.Dosim. 175, 313-320 (2017).

36. A. C. Turner, D. Zhang, M. Khatonabadi, M. Zankl, J. J. DeMarco, C. H. Cagnon, D. D. Cody, D. M. Stevens, C. H. McCollough, and M. F. McNittGray,"The feasibility of patient size-corrected, scanner-independent organ dose estimates for abdominal CT exams," Med. Phys. 38, 820-829 (2011). 